\title{
Biospecimen Usability
}

National Cancer Institute

\section{Source}

National Cancer Institute. Biospecimen Usability. NCI Thesaurus. Code C105758.

A qualitative or quantitative measure that describes a biospecimen's suitability for testing purposes. 